\title{
On Non-increasing of the Density and the Weak Density under Weakly Normal Functors of Finite Support
}

\author{
Beshimov R.B. ${ }^{1, *}$, Mamadaliev N.K. ${ }^{2}$ \\ ${ }^{1}$ Department of Mathematics, Tashkent State Pedagogical University named after Nizami, \\ str. Yusuf Khos Hojib 103, 100070 Tashkent, Uzbekistan \\ ${ }^{2}$ Institute of Mathematics, National University of Uzbekistan named after Mirzo Ulugbek, \\ Durmon yuli str. 29, 100125 Tashkent, Uzbekistan \\ *Corresponding Author: rbeshimov@mail.ru
}

Copyright (C)2015 by authors, all rights reserved. Authors agree that this article remains permanently open access under the terms of the Creative Commons Attribution License 4.0 International License

Abstract In the paper it is proved that if a covariant functor $F$ : Comp $\rightarrow$ Comp is weakly normal, then for any infinite Tychonoff space $X$ following inequalities hold: $d\left(F_{n}^{\beta}(X)\right) \leq d(X), d\left(F_{\omega}^{\beta}(X)\right) \leq d(X)$, $w d\left(F_{n}^{\beta}(X)\right) \leq w d(X), w d\left(F_{\omega}^{\beta}(X)\right) \leq w d(X)$.

Keywords Local Density, Local Weak Density, Probability Measure

AMS Subject Classification: 46E27, 54A25, 54C35

\section{Introduction}

Let $X$ be a $T_{1}$-space. The set of all nonempty closed subsets of a space $X$ denote by $\exp X$. The family of all sets in the form

$O\left\langle U_{1}, \ldots, U_{n}\right\rangle=\left\{F: F \in \exp X, F \subset \bigcup_{i=1}^{n} U_{i}, F \cap U_{i} \neq \emptyset, i=1,2, \ldots, n\right\}$, where $U_{1}, \ldots, U_{n}$ is a sequence of open sets in $X$, generates a topology on the set $\exp X$. This topology is called the Vietoris topology. The set exp $X$ with the Vietoris topology is called the exponential space or the hyperspace of $X$ [1].

Denote by $\exp _{n} X$ the family of all nonempty finite subsets of a space $X$, consisting of at most $\mathrm{n}$ elements, i.e. $\exp _{n} X=\{F \in \exp X:|F| \leq n\}$. Denote by $\exp _{\omega} X$ the family of all finite subsets of $X$. It is easy to see that $\exp _{\omega} X=\bigcup\left\{\exp _{n} X: n=1,2, \ldots n, \ldots\right\}$. Denote by $\exp _{c} X$ the family of all closed compact subsets of $X$.

In [1] it is shown that the functor exp : Comp $\rightarrow$ Comp is normal. In the work [2] it is proved that for any $T_{1}$-space $X$

$$
d(X)=d\left(\exp _{n} X\right)=d\left(\exp _{\omega} X\right)=d\left(\exp _{c} X\right)=d(\exp X)
$$

hold.

It is known from [3] that for any $T_{1}$-space $X$

$$
w d(X)=w d\left(\exp _{n} X\right)=w d\left(\exp _{\omega} X\right)=w d\left(\exp _{c} X\right)=w d(\exp X)
$$

hold.

From above mentioned statements it is natural to put following

1.1. Question. For which normal functors $F:$ Comp $\rightarrow$ Comp following equalities hold:

$$
\begin{gathered}
d(X)=d\left(F_{n}^{\beta}(X)\right)=d\left(F_{\omega}^{\beta}(X)\right)=d\left(F^{\beta}(X)\right), \\
w d(X)=w d\left(F_{n}^{\beta}(X)\right)=w d\left(F_{\omega}^{\beta}(X)\right)=w d\left(F^{\beta}(X)\right),
\end{gathered}
$$

where $F_{n}^{\beta}, F_{\omega}^{\beta}, F^{\beta}$ are functors in the category of all Tychonoff spaces and their continuous mappings. 
In the current work preserving of the density and the weak density by the functor of probability measures of finite support, is investigated.

Recall some necessary definitions.

A covariant functor $F: C o m p \rightarrow C o m p$ is normal, if it is continuous, preserves the weight, intersections and pre-images, is monomorphic, is epimorphic, transforms a one-point set and empty set to a one-point and empty set, respectively [1].

A covariant functor $F: C o m p \rightarrow C o m p$ is weakly normal, if it satisfies all conditions of normality except of preserving pre-images [4].

A functor $L:$ Tych $\rightarrow$ Tych is normal [5], if it is continuous, preserves the weight, embeddings, intersections, singletons, the empty set and translates $k$-covering mappings to surjections.

In the work [5] A.Ch.Chigogidze proved that if a functor $F: C o m p \rightarrow C o m p$ defined in the category of compacts and their continuous mappings, is normal, then it can be extended over the category of all Tychonoff spaces and their continuous mappings, preserving its normality. It is clear that for every normal functor $L: T y c h \rightarrow T y c h$, every $X \in O b(T y c h)$ and each point $x \in L(X)$ there exists (by property of preserving intersections) smallest subspace $A \subseteq X$ such that $x \in L(A)$. The closure of this space in $X$, is called the support of a point $x$, the set itself is the kernel of the support. A functor $L$ is a functor of finite support, if for any $X \in O b(T y c h)$ and for any point $x \in L(X)$ the support of $x$ is compact.

A continuous mapping $f: X \rightarrow Y$ is a $k$-covering mapping, if for any compact $B \subset Y$ there is a compact set $A \subset X$ such that $f(A)=B$. Every perfect mapping is $k$-covering [6].

Let $F: C o m p \rightarrow C o m p$ be an arbitrary normal functor and let $X \in O b($ Tych $)$. Put $F^{\beta}(X)=\{x \in F(\beta X)$ : $\operatorname{supp}(x) \subseteq X\}$. For a morphism $f: X \rightarrow Y$ in the category Tych put $F^{\beta}(f)=F(\beta f) \mid F^{\beta}(X)$, where $\beta f: \beta X \rightarrow \beta Y$ is the Stone-Czech extension of the mapping $f$. Using normality of the functor $F$ one can show that $F^{\beta}(f)$ is welldefined, i.e. $F(\beta f) F^{\beta}(X) \subseteq F^{\beta}(Y)$. It is easy to see that $F^{\beta}$ is a covariant functor in the category $T y c h$.

By $P_{n}$ denote the functor assigning to a compact space $X$ the set of all elements $a \in P(X)$, the support of which consists of at most $n$ points.

Let $X \in C o m p$. By $C(X)$ denote the set of all continuous mappings $\varphi: X \rightarrow R$ of a space $X$ with the usual sup-norm:

$$
\|\varphi\|=\sup \{|\varphi(x)|: x \in X\} .
$$

For each $c \in R$ by $c_{X}$ denote the constant function defined by the formula $c_{X}(x)=c, x \in X$. Suppose $\varphi, \psi \in C(X)$. An inequality $\varphi \leq \psi$ means that $\varphi(x) \leq \psi(x)$ for all $x \in X$.

A mapping $\nu: C(X) \rightarrow R$ is called a functional.

Definition 1.1[4]. A functional $\nu: C(X) \rightarrow R$ is called:

1) weakly additive if $\nu\left(\varphi+c_{X}\right)=\nu(\varphi)+c \cdot \nu\left(1_{X}\right)$ for all $c \in R$ and $\varphi \in C(X)$;

2 ) order-preserving, if for functions $\varphi, \psi \in C(X)$ from $\varphi \leq \psi$ it follows $\nu(\varphi) \leq \nu(\psi)$;

3) normed if $\nu\left(1_{X}\right)=1$.

For a compact $X$ by $O(X)$ denote the set of all weakly additive order-preserving normed functionals. Elements of the set $O(X)$, are shortly called weakly additive functionals. Note that each functional $\nu \in O(X)$ is a continuous mapping of $C(X)$ to $R$, i.e. the set $O(X)$ is a subset of $C_{p}(C(X))$. The set is equipped with the pointwise topology. Sets in the form

$$
\left\langle\mu ; \varphi_{1}, \ldots, \varphi_{k} ; \varepsilon\right\rangle=\left\{\nu \in O S(X):\left|\mu\left(\varphi_{i}\right)-\nu\left(\varphi_{i}\right)\right|<\varepsilon, i=1, \ldots, k\right\}
$$

where $\varphi_{i} \in C(X), i=1, \ldots, k, \varepsilon>0$, generates a neighborhood base of a functional $\mu$ in $O(X)$.

Let $X, Y \in C o m p$ and let $f: X \rightarrow Y$ be a continuous mapping. Define the mapping $O(f): O(X) \rightarrow O(Y)$ getting

$$
(O(f)(\mu))(\varphi)=\mu(\varphi \circ f)
$$

where $\mu \in O(X)$ and $\varphi \in C(Y)$.

In [4] T.Radul proved that the covariant functor $O:$ Comp $\rightarrow$ Comp of weakly additive order-preserving normed functionals in the category of compacts, satisfies all conditions of normality except pre-image preserving.

A positive normed measure is called a probability measure. A space consisting of all probability measures, denote by $P(X)$. A neighborhood base at a point $\mu \in P(X)$ consists of all the sets in the form

$$
O\left(\mu ; \phi_{1}, \phi_{2}, \ldots, \phi_{k} ; \varepsilon\right)=\left\{\nu \in P(X):\left|\mu\left(\phi_{i}\right)-\nu\left(\phi_{i}\right)\right|<\varepsilon, i=1,2, \ldots, k\right\}
$$

where $\phi_{1}, \phi_{2}, \ldots, \phi_{k} \in C(X)$ and $\varepsilon>0$.

A support $\operatorname{supp}(\mu)$ of a measure $\mu \in P(X)$ is the smallest closed subset $F \subset X$ such that $\mu(F)=\mu(X)$. For a compact $X$ and a natural number $n$ put $P_{n}(X)=\{\mu \in P(X):|\operatorname{supp}(\mu)| \leq n\}[1]$.

\section{Main Results}

2. The density and the weak density of the space of probability measures of finite support in the category of Tychonoff spaces.

In this chapter we shall prove that the functor of probability measures of finite support preserves the density and the weak density in the category of compacts. 
Theorem 2.1. For an arbitrary infinite compact space $X$ we have

$$
d(X)=d\left(P_{n}(X)\right) \text {. }
$$

Proof. Let's prove first $d\left(P_{n}(X)\right) \leq d(X)$. Suppose $X$ is an infinite compact and $d(X)=\tau \geq \aleph_{0}$. It is clear that $d\left(X^{n}\right)=\tau$ for every $n \in N$. By Basmanov's theorem ([7]) we see that the space $P_{n}(X)$ can be represented as the continuous image of the product $X^{n} \times \sigma^{n-1}$, where $\sigma^{n-1}$ is $(n-1)$-dimensional simplex. The mapping $\pi: X^{n} \times \sigma^{n-1} \rightarrow P_{n}(X)$ is defined by the formula:

$$
\pi\left(x_{1}, \ldots, x_{n}, m_{1}, \ldots, m_{n}\right)=\sum_{i=1}^{n} m_{i} \delta_{x_{i}}
$$

where $\left(m_{1}, \ldots, m_{n}\right) \in \sigma^{n-1}, \sum_{i=1}^{n} m_{i}=1$ and $m_{i} \geq 0$ for every $i \in N, \delta_{x_{i}}$ are Dirac measures at points $x_{i}$, respectively. Hence, $d\left(X^{n} \times \sigma^{n-1}\right) \leq \tau$ and from the fact that the density of the space is preserved under continuous mappings, we obtain $d\left(P_{n}(X)\right) \leq \tau$.

Let us now prove that $d(X) \leq d\left(P_{n}(X)\right)$. Let $d\left(P_{n}(X)\right)=\tau \geq \aleph_{0}$. Then there is a dense subset $\Omega=\left\{\mu_{\alpha}: a \in A\right\}$ of $P_{n}(X)$ such that $|A|=\tau$. We construct the subset $M$ of $X$ with the following way:

$M=\bigcup_{\alpha \in A}\left\{\operatorname{supp}\left(\mu_{\alpha}\right):\left|\operatorname{supp}\left(\mu_{\alpha}\right)\right| \leq n, \mu_{\alpha} \in \Omega\right\}$. Clearly, $|M|=\tau$. We have to show that $M$ is dense in $X$. Suppose it doesn't hold, i.e. there is a point $x_{0}$ and its neighborhood $O x_{0}$ such that $M \cap O x_{0}=\emptyset$. By regularity of $X$ there exists a neighborhood $O_{1} x_{0}$ of the point $x_{0}$ such that $\left[O_{1} x_{0}\right] \subset O x_{0}$. Since $X$ is normal, there is a function $\varphi \in C(X)$ such that $\varphi\left(\left[O_{1} x_{0}\right]\right)=0$ and $\varphi\left(X \backslash O x_{0}\right)=1$.

Consider the neighborhood $O\left(\delta_{x_{0}} ; \varphi ; 0,5\right)$ of the point $\delta_{x_{0}}$ in $P_{n}(X)$. Since $\Omega$ is dense in $P_{n}(X)$, there exists an element $\nu \in \Omega \bigcap O\left(\delta_{x_{0}} ; \varphi ; 0,5\right)$. Let $\sup p(\nu)=\left\{x_{1}, x_{2}, \ldots, x_{k}\right\}, k \leq n$. Then

$$
\left|\nu(\varphi)-\delta_{x_{0}}(\varphi)\right|=\left|\sum_{i=1}^{k} m_{i} \delta_{x_{i}}(\varphi)-\delta_{x_{0}}(\varphi)\right|=\left|\sum_{i=1}^{k} m_{i} \varphi\left(x_{i}\right)-\varphi\left(x_{0}\right)\right|=
$$

$=\sum_{i=1}^{k} m_{i}=1>0,5$.

This contradiction to density of the set $\Omega$ in $P_{n}(X)$ shows that the set $M$ is dense in $X$, i.e. $d(X) \leq \tau$. Theorem 2.1 is proved.

Theorem 2.2. For any infinite compact $X$

$$
d(X)=d\left(P_{\omega}(X)\right)
$$

holds.

Proof. We shall prove first $d\left(P_{\omega}(X)\right) \leq d(X)$. Let $X$ be an infinite compact and let $d(X)=\tau \geq \aleph_{0}$. It is clear that $d\left(X^{n}\right)=\tau$ for every $n \in N$. Then $d\left(P_{n}(X)\right) \leq \tau$ for every $n \in N$ by theorem 2.1. Since $P_{\omega}(X)=\bigcup\left\{P_{n}(X)\right.$ : $n=1,2, \ldots\}$, we have $d\left(\cup\left\{P_{n}(X): n=1,2, \ldots\right\}\right)=d\left(P_{\omega}(X)\right) \leq \tau$.

Let us now prove that $d(X) \leq d\left(P_{\omega}(X)\right)$. Suppose $d\left(P_{\omega}(X)\right)=\tau \geq \aleph_{0}$. Then there exists a dense subset $\Omega=\left\{\mu_{\alpha}: a \in A\right\}$ of $P_{\omega}(X)$, where $|A|=\tau$. Let us construct the subset $M$ of $X$ with the following way:

$$
M=\bigcup_{\alpha \in A}\left\{\operatorname{supp}\left(\mu_{\alpha}\right):\left|\operatorname{supp}\left(\mu_{\alpha}\right)\right|<\infty, \mu_{\alpha} \in \Omega\right\} .
$$

It is obvious that $|M|=\tau$. We shall show that $M$ is dense in $X$. Suppose that $M$ is not dense in $X$. Then there is a point $x_{0}$ and its neighborhood $O x_{0}$ such that $M \bigcap O x_{0}=\emptyset$. By regularity of $X$ there exists a neighborhood $O_{1} x_{0}$ of the point $x_{0}$ such that $\left[O_{1} x_{0}\right] \subset O x_{0}$. Since $X$ is normal, there is a function $\varphi \in C(X)$ such that $\varphi\left(\left[O_{1} x_{0}\right]\right)=0$ and $\varphi\left(X \backslash O x_{0}\right)=1$.

Consider the neighborhood $O\left(\delta_{x_{0}} ; \phi ; \frac{1}{4}\right)$ of the point $\delta_{x_{0}}$ in $P_{\omega}(X)$. Since $\Omega$ is dense in $P_{\omega}(X)$, there is the intersection is not empty, i.e. there is an element $\nu \in \Omega \cap O\left(\delta_{x_{0}} ; \phi ; \frac{1}{4}\right)$. Let $\operatorname{supp}(\nu)=\left\{x_{1}, x_{2}, \ldots, x_{n}\right\}$. Then

$$
\left|\nu(\phi)-\delta_{x_{0}}(\phi)\right|=\left|\sum_{i=1}^{n} m_{i} \delta_{x_{i}}(\phi)-\delta_{x_{0}}(\phi)\right|=\left|\sum_{i=1}^{n} m_{i} \phi\left(x_{i}\right)-\phi\left(x_{0}\right)\right|=1>\frac{1}{4} .
$$

This contradicts density of the set $\Omega$ in $P_{\omega}(X)$. Hence the set $M$ is dense in $X$. This implies $d(X) \leq \tau$. Theorem 2.2 is proved.

From theorems 2.1 and 2.2 we obtain the following

Corollary 2.1. For any infinite compact $X$ and any $n \in N$ we have

$$
d(X)=d\left(P_{n}(X)\right)=d\left(P_{\omega}(X)\right) .
$$

3. Density and weak density of the space of probability spaces in the category of Tychonoff spaces. In this chapter preserving of the density and the weak density by weakly normal functors of finite support, is investigated.

We need following definition and theorems. 
Definition 3.1 [9]. The weak density of a topological space $X$ is the smallest cardinal number $\tau \geq \aleph_{0}$ such that there is a $\pi$-base in $X$ coinciding with $\tau$ centered systems of open sets, i.e. there is a $\pi$-base $\mathrm{B}=\bigcup\left\{B_{\alpha}: \alpha \in A\right\}$, where $B_{\alpha}$ is a centered system of open sets for each $\alpha \in A$ and $|A|=\tau$.

The weak density of a topological space $X$ is denoted by $w d(X)$. If $w d(X)=\aleph_{0}$ then we say that a topological space $X$ is weakly separable [10].

Theorem 3.2 [8]. If $Y$ is dense in $X$, then $w d(Y)=w d(X)$.

Theorem 3.3 [11]. Let $P$ be a functor of probability measures. Then $P_{\omega}(X)$ is dense in the space $P(X)$ for any compact $X$.

The following proposition can be easily obtained from [12].

Proposition 3.1. Let $X$ be an infinite Tychonoff space, $b X$ its arbitrary compact extension. Then for any natural number $n \in N$ the space $P_{n}(X)$ is dense in the space $P_{n}(b X)$.

Proposition 3.2. Suppose that $X$ and $Y$ are Tychonoff spaces such that $X$ is a dense subspace of $Y$. Then $P_{\omega}^{\beta}(X)$ is dense subspace of $P_{\omega}^{\beta}(Y)$.

Proposition 3.3. Let $X$ be an infinite Tychonoff space, $b X$ its arbitrary compact extension. Then $P_{\omega}^{\beta}(X)$ is a dense subspace of $P(b X)$.

Proposition 3.4. Let $X$ be an infinite Tychonoff space. If $X$ is a dense subspace of a Tychonoff space $Y$, then $P_{\omega}^{\beta}(X)$ is dense in $P^{\beta}(Y)$.

Proof. Assume that $X$ is a dense subspace of a Tychonoff space $Y$. Then $X$ is dense in $\beta Y$. By proposition 3.3 the set $P_{\omega}^{\beta}(X)$ is dense in the compact $P(\beta Y)$. But $P_{\omega}^{\beta}(X) \subset P^{\beta}(Y) \subset P(\beta Y)$. Hence $P_{\omega}^{\beta}(X)$ is dense in $P^{\beta}(Y)$. Proposition 3.4 is proved.

Theorem 3.4. Let $X$ be an infinite Tychonoff space and let $n \in N$. Then

$$
w d(X)=w d\left(P_{n}(X)\right)=w d\left(P_{\omega}^{\beta}(X)\right)=w d\left(P^{\beta}(X)\right) .
$$

Proof. Let $X$ be an infinite Tychonoff space and let $w d(X)=\tau \geq \aleph_{0}$. Then by theorem 3.2[8] for an arbitrary compact extension $b X$ of $X$ we have $w d(X)=d(b X)=\tau$. By corollary 2.1 we obtain

$$
w d(X)=d(b X)=d\left(P_{n}(b X)\right)=d\left(P_{\omega}(b X)\right)=\tau
$$

Since spaces $b X$ and $P_{n}(b X)$ are compact, by propositions 3.1 and 3.2 we see that the space $P_{n}(X)$ is dense in $P_{n}(b X)$, the space $P_{\omega}^{\beta}(X)$ is dense in $P_{\omega}^{\beta}(b X)$. Therefore, $P_{\omega}^{\beta}(X)$ is dense in $P^{\beta}(X)$. It is known that the weak density is hereditary with respect to open subspaces and compact extensions. Therefore, from theorems $4[9]$ and $2.3[10]$ it follows

Theorem 3.4 is proved.

$$
w d(X)=w d\left(P_{n}(X)\right)=w d\left(P_{\omega}^{\beta}(X)\right)=w d\left(P^{\beta}(X)\right)=\tau .
$$

Theorem 3.5. If a covariant functor $F: C o m p \rightarrow C o m p$ is weakly normal, then for an arbitrary infinite Tychonoff space $X$ we have

$$
\begin{aligned}
& \text { 1. } d\left(F_{n}^{\beta}(X)\right) \leq d(X) ; \\
& \text { 2. } d\left(F_{\omega}^{\beta}(X)\right) \leq d(X) .
\end{aligned}
$$

Proof. 1) Let $Y$ be a dense subspace of a Tychonoff space $X$ such that $|Y|=\tau=d(X)$. We have $\left|\exp _{n} Y\right|=\tau$, because $Y$ is infinite. Since $F$ preserves weight, for any finite compact $F(Z)=F^{\beta}(Z)$ is metrizable, and therefore, is separable. For each $Z \in \exp _{n} Y$ fix a countable dense subset $D_{Z}$ of $F(Z)$. Since all sets $D_{Z}$ are countable, we have $|D| \leq \omega_{0} \cdot\left|\exp _{n} Y\right|=\tau$, where $D=\cup\left\{D_{Z}: Z \in \exp _{n} Y\right\}$. It is clear that $D$ is dense in the set $\cup\left\{F(Z): Z \in \exp _{n} Y\right\}=F_{n}(Y)=F_{n}^{\beta}(Y)$.

Let $b X$ be an arbitrary compact extension of the space $X$. By lemma $2.1[8]$ the set $F_{n}^{\beta}(Y)$ is dense in the set $F_{n}^{\beta}(X)$. Consequently, the set $D$ is dense in $F_{n}^{\beta}(X)$. Therefore $d\left(F_{n}^{\beta}(X)\right) \leq d(X)$.

2) Let $Y$ be a dense subset of a Tychonoff space $X$ such that $|Y|=\bar{\tau}=d(X)$. Since $Y$ is infinite, we have $\left|\exp _{\omega} Y\right|=\tau$. By the fact that $F$ preserves weight, for any finite $Z$ the compact $F(Z)=F^{\beta}(Z)$ is metrizable, and therefore, is separable. For each $Z \in \exp _{\omega} Y$ fix a countable dense subset $D_{Z}$ of $F(Z)$. Since all sets $D_{Z}$ are countable, we have $|D| \leq \omega_{0} \cdot\left|\exp _{\omega} Y\right|=\tau$, where $D=\cup\left\{D_{Z}: Z \in \exp _{\omega} Y\right\}$. It is clear that $D$ is dense in the set $\cup\left\{F(Z): Z \in \exp _{\omega} Y\right\}=F_{\omega}(Y)=F_{\omega}^{\beta}(Y)$.

Let $b X$ be an arbitrary compact extension of the space $X$. By proposition $2.1[8]$ the set $F_{\omega}^{\beta}(Y)$ is dense in the set $F_{\omega}^{\beta}(X)$. Consequently, the set $D$ is dense in $F_{\omega}^{\beta}(X)$. Hence $d\left(F_{\omega}^{\beta}(X)\right) \leq d(X)$. Theorem 3.5 is proved.

Corollary 3.1. If a covariant functor $F: C o m p \rightarrow C o m p$ is weakly normal, then for any infinite Tychonoff space $X$ we have

$$
\begin{aligned}
& \text { 1) } d\left(F_{n}^{\beta}(X)\right) \leq d(X) ; \\
& \text { 2) } d\left(F_{\omega}^{\beta}(X)\right) \leq d(X) ; \\
& \text { 3) } d\left(F^{\beta}(X)\right) \leq d(X) .
\end{aligned}
$$

Corollary 3.2. If a covariant functor $F:$ Comp $\rightarrow$ Comp is weakly normal, then for any infinite separable space $X$ spaces $F_{n}^{\beta}(X), F_{\omega}^{\beta}(X), F^{\beta}(X)$ are separable too. 
Theorem 3.6. If a covariant functor $F: C o m p \rightarrow C o m p$ is weakly normal, then for any infinite Tychonoff space $X$ we have

$$
\begin{aligned}
& \text { 1) } w d\left(F_{n}^{\beta}(X)\right) \leq w d(X) \text {; } \\
& \text { 2) } w d\left(F_{\omega}^{\beta}(X)\right) \leq w d(X) \text {; } \\
& \text { 3) } w d\left(F^{\beta}(X)\right) \leq w d(X) .
\end{aligned}
$$

Proof. Let $X$ be an infinite Tychonoff space and let $w d(X)=\tau \geq \aleph_{0}$. Then by results from [13] we have $d(\beta X)=\tau$. From theorem 3.5 it follows $d\left(F_{n}^{\beta}(X)\right) \leq d(X) ; d\left(F_{\omega}^{\beta}(X)\right) \leq d(X) ; d\left(F^{\beta}(X)\right) \leq d(X)$. It is known that inequalities $c(X) \leq w d(X) \leq d(X)[8]$ hold for any topological space. Therefore we obtain inequalities $w d\left(F_{n}^{\beta}(X)\right) \leq w d(X), w d\left(F_{\omega}^{\beta}(X)\right) \leq w d(X), w d\left(F^{\beta}(X)\right) \leq w d(X)$. Theorem 3.6 is proved.

Corollary 3.3. If a covariant functor $F: \operatorname{Comp} \rightarrow$ Comp is weakly normal, then for any infinite weakly separable space $X$ spaces $F_{n}^{\beta}(X), F_{\omega}^{\beta}(X), F^{\beta}(X)$ are weakly separable.

\section{Conclusion}

In the future works, we are planning to investigate cardinal invariants and functors preserving them. Now, we are trying to give answers to the following open questions: Does the functor $F$ preserve the tightness, the density, the weak density, the local density and the local weak density, where $F$ can be the exponential functor exp, the functor of superextension $\lambda$, the functor of probability measures $P$, the functor of weakly additive functionals $O$ and the functor of semi-additive functionals $O S$ ?

\section{Acknowledgements}

We would like to thank to reviewers for their helpful advices and detailed comments that helped to increase the quality of the paper.

\section{REFERENCES}

[1] Fedorchuk V.V., Filippov V.V. General topology. Basic constructions. Moscow: Fizmatlit, 2006, 332 p.

[2] Michael. E. Topologies on spaces of supsets Trans.Amer.Math.Soc. - 1951. - No 1 (71). - P. 152-172.

[3] Beshimov R.B. On some cardinal invariants of hyperspaces Mathematychni Studii. - 2005. No 2 (24). p. $197-202$.

[4] Radul T. N. On the functor of order-preserving functionals Comment. Math.Univ. Carol. - 1998. - No 3 (39). - p. 609-615.

[5] Chigogidze A.Ch. On extensions of normal functors. Vestnik MSU. Ser. math.-mech. -1984. -No 6. -p.23-26.

[6] Shapiro L.B. On operators of extension of functions and normal functors. Vestnik MSU Ser. math.-mech. -1992. -No 1. - p.35-42.

[7] Basmanov V.N. Covariants functors, retracts and dimension. DAN USSR. -1983. -No 5 (271). -p.1033-1036.

[8] Beshimov R.B. Some cardinal properties of topological spaces connected with weakly density Methods of Functional Analysis and Topology. - 2004. - No 3 (10). p. 17-22.

[9] Beshimov R.B., On weakly density of topological spaces. DANRUz, 2000 No 11, p. 10-13.

[10] Beshimov R.B. O note weakly separable spaces Mathematica Moravica. - 2002. - (6). - p. 9-19.

[11] Fedorchuk V.V. On some geometrical properties of covariant functors. UMN. -1984. -No 2(39). -p.169-208.

[12] Beshimov R.B. On non-increasing of density and weak density under weakly normal functors. Mathematical Notes, 2008, V.84, No 4, p.527-531.

[13] van Douwen E.K. Density of compactifications Set-theoretic topology. N.Y.Acad. Press. - 1977. - P. 97-110. 